OPEN ACCESS

Edited by: Mitali Dandekar,

Paras Cancer Centre, India

Reviewed by:

Wiktoria Maria Suchorska Greater Poland Cancer Center

(GPCC), Poland

Derfel Ap Dafydd,

Royal Marsden Hospital,

United Kingdom

*Correspondence:

Fada Xia

xiafada@csu.edu.cn

Specialty section:

This article was submitted to

Head and Neck Cancer,

a section of the journal

Frontiers in Oncology

Received: 28 February 2021 Accepted: 17 September 2021

Published: 14 October 2021

Citation:

Zhang Z, Yao L, Wang W, Jiang $B$, $X i a$ F and Li X (2021) A Bibliometric Analysis of 34,692 Publications on Thyroid Cancer by Machine Learning: How Much Has Been Done in the Past Three Decades?

Front. Oncol. 11:673733. doi: 10.3389/fonc. 2021.673733

\section{A Bibliometric Analysis of 34,692 Publications on Thyroid Cancer by Machine Learning: How Much Has Been Done in the Past Three Decades?}

\author{
Zeyu Zhang, Lei Yao, Wenlong Wang, Bo Jiang, Fada Xia * and Xinying Li \\ Department of Thyroid Surgery, Xiangya Hospital, Central South University, Changsha, China
}

Introduction: Thyroid cancer (TC) is the most common neck malignancy. However, a large number of publications of TC have not been well summarized and discussed with more comprehensive methods. The purpose of this bibliometric study is to summarize scientific publications during the past three decades in the field of TC using a machine learning method.

Material and Methods: Scientific publications focusing on TC from 1990 to 2020 were searched in PubMed using the MeSH term "thyroid neoplasms". Full associated data were downloaded in the format of PubMed, and extracted in the R platform. Latent Dirichlet allocation (LDA) was adopted to identify the research topics from the abstract of each publication using Python.

Results: A total of 34,692 publications related to TC from the last three decades were found and included in this study with an average of 1,119.1 publications per year. Clinical studies and experimental studies shared the most proportion of publications, while the proportion of clinical trials remained at a relatively small level $(5.87 \%$ as the highest in 2004). Thyroidectomy was the lead MeSH term, followed by prognosis, differential diagnosis, and fine-needle biopsy. The LDA analyses showed the study topics were divided into four clusters, including treatment management, basic research, diagnosis research, epidemiology, and cancer risk. However, a relatively weak connection was shown between treatment managements and basic researches. Top 10 most cited publications in recent years particularly highlighted the applications of active surveillance in TC.

Conclusion: Thyroidectomy, differential diagnosis, genomic analysis, active surveillance are the most concerning topics in TC researches. Although the BRAF-targeted therapy is under development with promising results, there is still an urgent need for conversions from basic studies to clinical practice.

Keywords: thyroid cancer, bibliometrics, machine learning, natural language processing, latent Dirichlet allocation 


\section{INTRODUCTION}

Thyroid cancer (TC) is the most common neck malignancy and is on the rise to become the fourth leading type of cancer worldwide (1). During the past decades, great progress and significant developments have been achieved in the field of TC with a growing number of publications. These scientific publications can reflect the hotspots of researches and suggest the future research direction (2). A bibliometric analysis is often used to quantify the progress of the field through published literature. The most recent bibliometric analyses by Cooper et al. summarized 19,055 articles from 2006 to 2015 in the broad area of clinical thyroid disease (3). However, the large amount of publication data in the special field of TC have not been well summarized and discussed with more comprehensive methods.

Natural language processing (NLP) is a series of machine learning methods for human language analyses. Among these methods, the Latent Dirichlet allocation (LDA) is most usually used to analyze scientific publications by identifying specific themes and dividing documents into these themes (4-6). In addition, LDA has been used in bibliometric analyses in the field of rectal cancer and gliomas $(7,8)$.

The present study is aiming at summarizing scientific publications of TC in the past three decades. Moreover, by detailedly analyzing the research topics using a machine learning method, we are hoping to provide insights into scientific developments, recognize areas with declining interest, and more importantly, reveal potential future research foci in the field of TC.

\section{MATERIALS AND METHODS}

Scientific publications focusing on TC from 1990 to 2020 were searched in PubMed using the MeSH term "thyroid neoplasms" on January 26, 2021, limited by the language of English. Full associated data were downloaded in the format of PubMed, and extracted in the R platform using the package "Bibliometrix", (9) including the publication year, the publication type, $\mathrm{MeSH}$ terms, abstract, etc. MeSH terms appearing more than 25 times were included in analyses. In addition, the present study did not need ethical approval from an institutional review board or ethics committee because this was a bibliometric analysis.

LDA was adopted to identify the research topics from the abstract of each publication using Python. A feature glossary of terms was created by LDA according to the frequency of coexistence of vocabulary words in the publication series. Subsequently, LDA would calculate the most two probable research topics of each publication, depending on the frequency of appearance of these glossary words in each publication. In order to further investigate the relationship between these topics, a cluster analysis was performed using the Louvain algorithm.

The visualizations were achieved by R platform and Excel, while the network was constructed by Gephi (https://gephi.org/) (10). All the mentioned codes, including R code and Python code, were available on GitHub (https://github.com/yan-wen0614/MedicineBibliometric-Analysis).

\section{RESULTS}

A total of 34,692 publications related to TC from the last three decades were found and included in this study with an average of 1,119.1 publications per year. As shown in Figure 1A, the quantity of TC publications continuously raised during the past three decades and was plateauing since 2014. However, it declined to 937 in 2020, where the pandemic of COVID-19 may take the responsibility. The distribution of publication types is shown in Figure 1B. Clinical studies and experimental studies shared the most proportion of publications. With the development of evidence-based medicine, the proportion of meta-analysis gradually increased during these years, while the proportion of clinical trials remained at a relatively small level $(5.87 \%$ as the highest in 2004). Moreover, the country-scientific production is shown in Figure 3. China, USA, Italy, South Korea, and Japan were the top five countries producing TC-related publications (Figure 2). In addition, the top 10 affiliations with the highest scientific production are listed in Table $\mathbf{1}$.

\section{MeSH Term Analyses}

A total of 1,595 MeSH terms with 3,459,152 times of occurrence were included in detailed $\mathrm{MeSH}$ term analyses.

$\mathrm{MeSH}$ terms concerning the age of the study population were initially investigated (Figure 3). Eight age groups were identified as follows: infant (birth to $2-3$ months), preschool child ( $2-5$ years), child (6-12 years), adolescent (13-18 years), adult (19-44 years), middle-aged (45-64 years), aged (65-79 years), and aged (80 years and over). Overall, the middle-aged was the most predominant cohort in TC studies, as 536.94 occurrence times per year.

Figure 4A shows the top $10 \mathrm{MeSH}$ terms, representing the most concerned research foci. Thyroidectomy was the lead term, followed by prognosis, differential diagnosis, fine-needle biopsy, etc. In 2014, the researches focusing on prognosis surpassed the researches focusing on differential diagnosis, suggesting the focus of TC researches might move to treatment outcomes from the diagnosis of TC. Notably, fine-needle biopsy firstly appeared in 2000 , however rapidly growing as the third hot field in 2020 .

In order to acknowledge the role of TC treatments, we further identified the top $10 \mathrm{MeSH}$ terms associated with TC treatments except for thyroidectomy (Figure 4B). Iodine radioisotopes was the lead term of TC treatments, followed by combined modality therapy, neck dissection, adjuvant radiotherapy, etc. Among them, molecular targeted therapy, including sorafenib, and ablation therapy appeared after 2003. Additionally, iodine radioisotopes and neck dissection were the fastest growing fields.

\section{LDA Analyses}

A total of 21,491 publications were further analyzed using the LDA method, excluding publications without an abstract. Using 


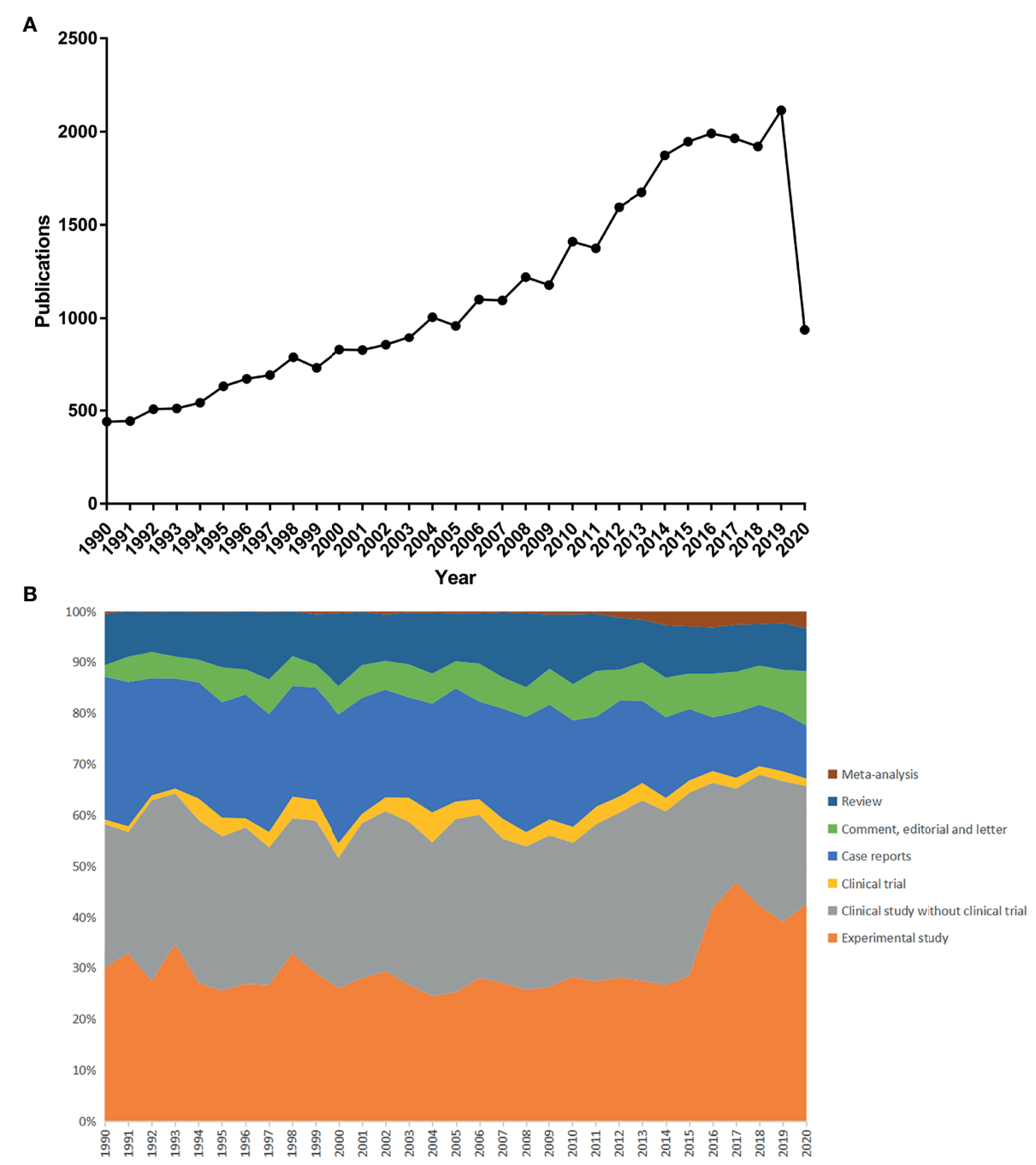

FIGURE 1 | (A) Scientific publications per year; (B) Distribution of publication types per year.

the text of the abstract, LDA analyses provided further information about hot topics and their relationships by constructing a topic network (Figure 5). The network was divided into four clusters, including treatment management (in red), basic research (in green), diagnosis research (in purple), and epidemiology and cancer risk (in blue). The focalization of topics and the weight of connections between topics were also shown as the size of the circle and the thickness of the line, respectively.

In the cluster of treatment management, tumor recurrence, lymph node invasion, and survival analysis were the top three studied topics. Great efforts were also made in the field of postoperative complications, radioiodine therapy, positron emission tomography/computed tomography (PET/CT) scan, and ablation therapy. In the cluster of diagnosis research, differential diagnosis, cytology of fine-needle aspiration (FNA), and ultrasound-guided fine needle aspiration biopsy
(US-FNAB) were the top three studied topics. Strong connections were shown between these two clusters, especially between differential diagnosis and tumor recurrence. The cluster of epidemiology and cancer risk covered a relatively small proportion of topics, however having high connectivity with the cluster of treatment management and the cluster of diagnosis research. Moreover, cancer cell proliferation and apoptosis, BRAF mutation, expression analyses of cell lines and tissues accounted for the main part of the basic research cluster. Notably, the topic of follicular adenomas and follicular thyroid carcinoma (FTC) also had a great significance in the basic research cluster, which was strongly connected with the cluster of diagnosis research. However, the rest of the basic research cluster showed a relatively poor connection with other clusters, suggesting that there may be an urgent need for conversions from basic researches to clinical treatments. 


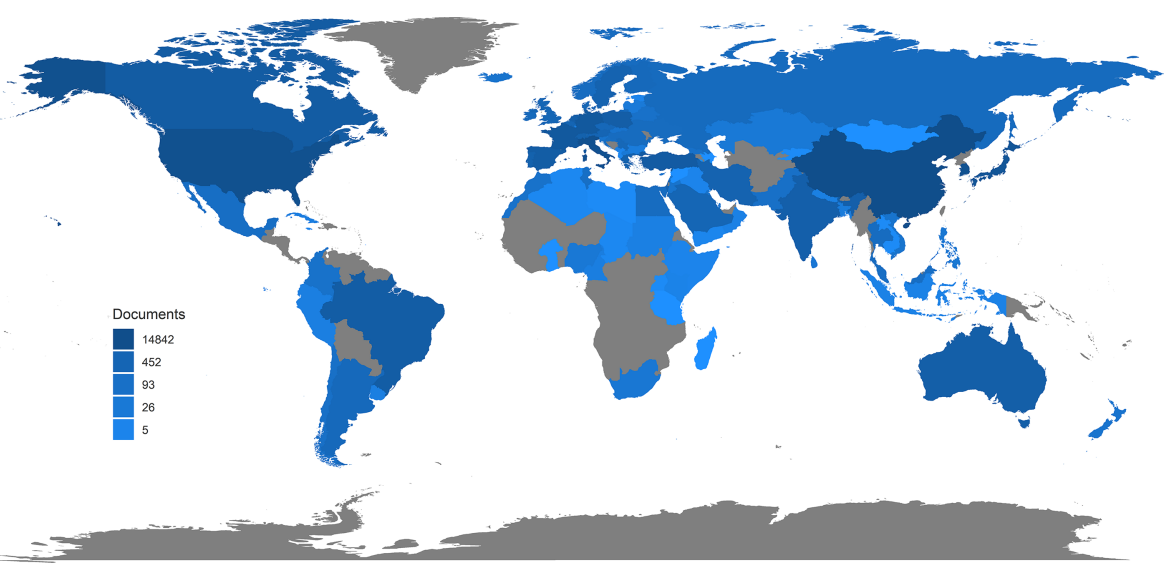

FIGURE 2 | Country-scientific production.

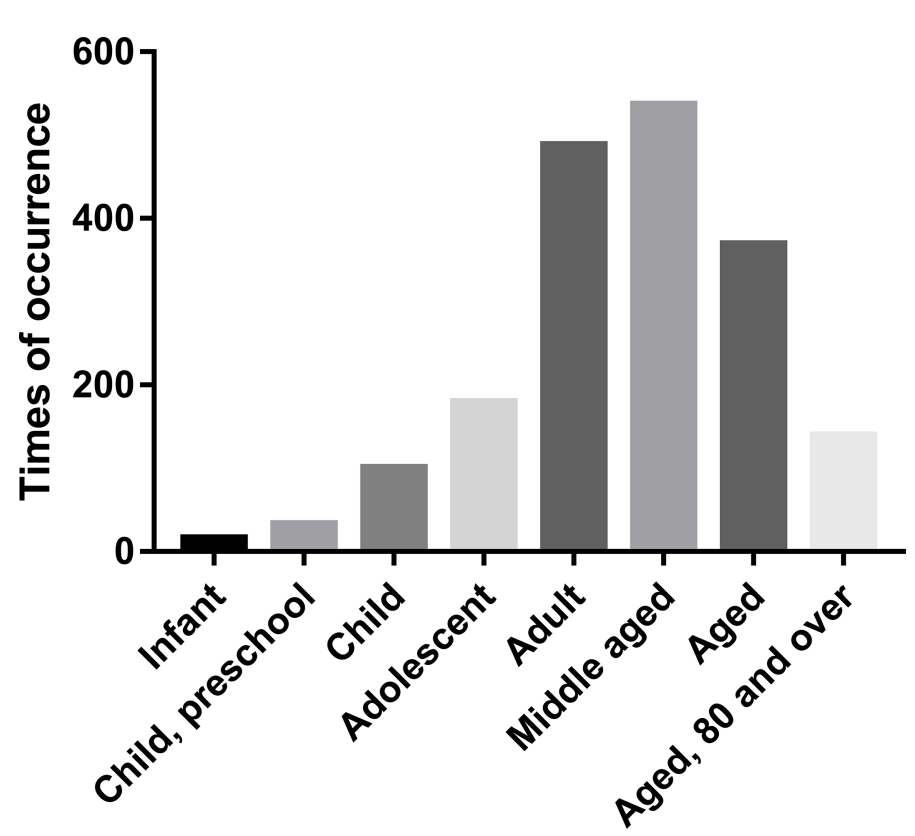

FIGURE 3 | The mean times of occurrence of MeSH terms concerning age per year.

TABLE 1 | Top 10 affiliations with highest scientific production.

Name of affiliation

University Of Ulsan College of Medicine

Yonsei University College of Medicine

University of Pisa

Sungkyunkwan University School of Medicine

Memorial Sloan Kettering Cancer Center

National Cancer Institute

Harvard Medical School

Fudan University

Kuma Hospital

Tongji University School of Medicine
Number of articles

1,032

964

876

739

664

657

638

630

564

508

\section{Top-Cited Publications}

Additional attention was put on recent top-cited publications. The top 10 most cited original researches from 2016 to 2020 were searched, while review, guidelines, and epidemiological papers were excluded. The results are listed in Table 2. Among these papers, three (1st, 5th, 7th) were concerning the overtreatment and active surveillance; two (2nd, 8th) were concerning the genomic and transcriptomic analyses; three (3rd, 6th, 9th) were concerning the surgical procedures; one (4th) was concerning the differential diagnosis, while one (10th) was concerning the targeted therapy of advanced TC. 


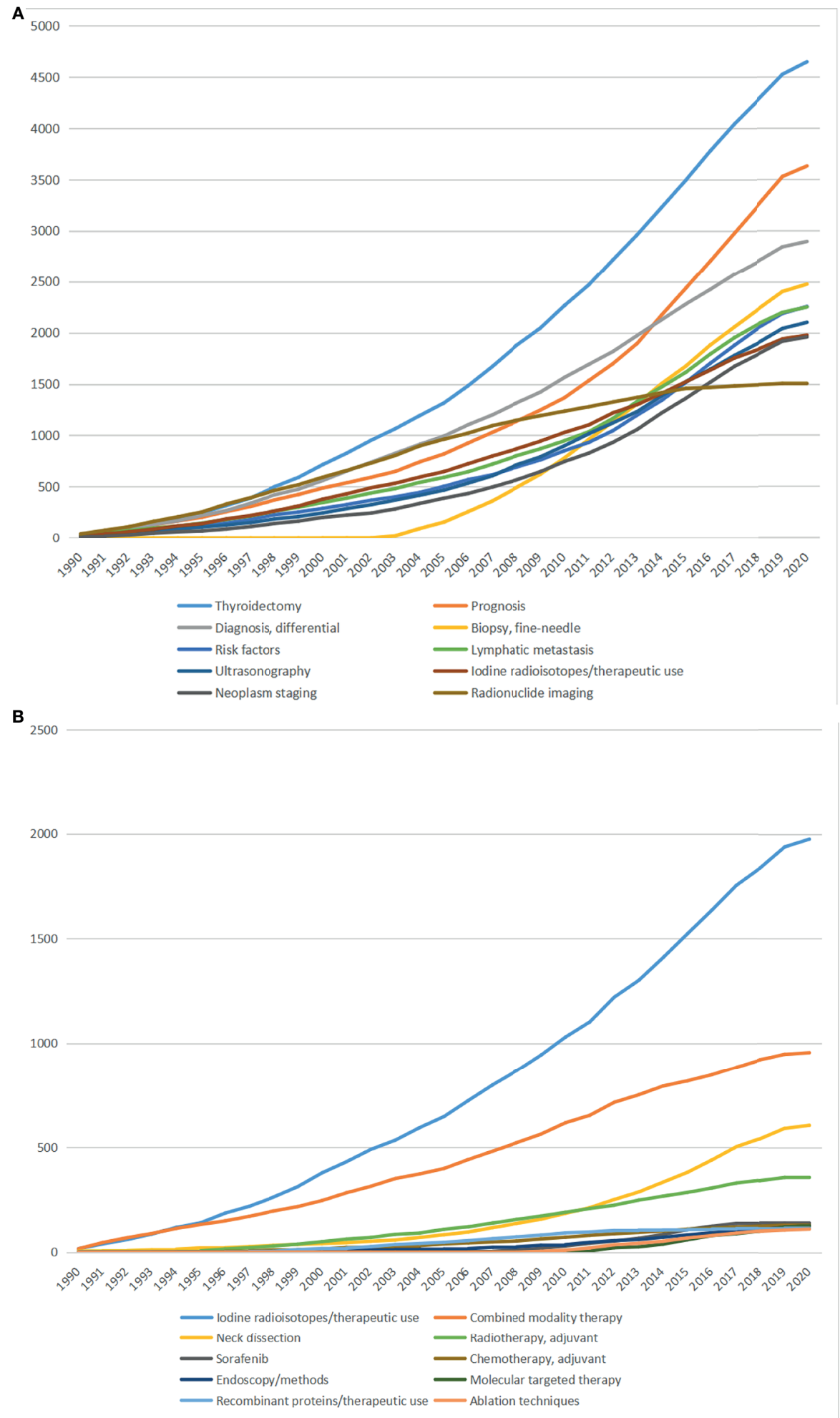

FIGURE 4 | (A) Top 10 MeSH terms and accumulative occurrences during the past three decades; (B) Top 10 MeSH terms concerning treatments of thyroid cancer and accumulative occurrences during the past three decades. 


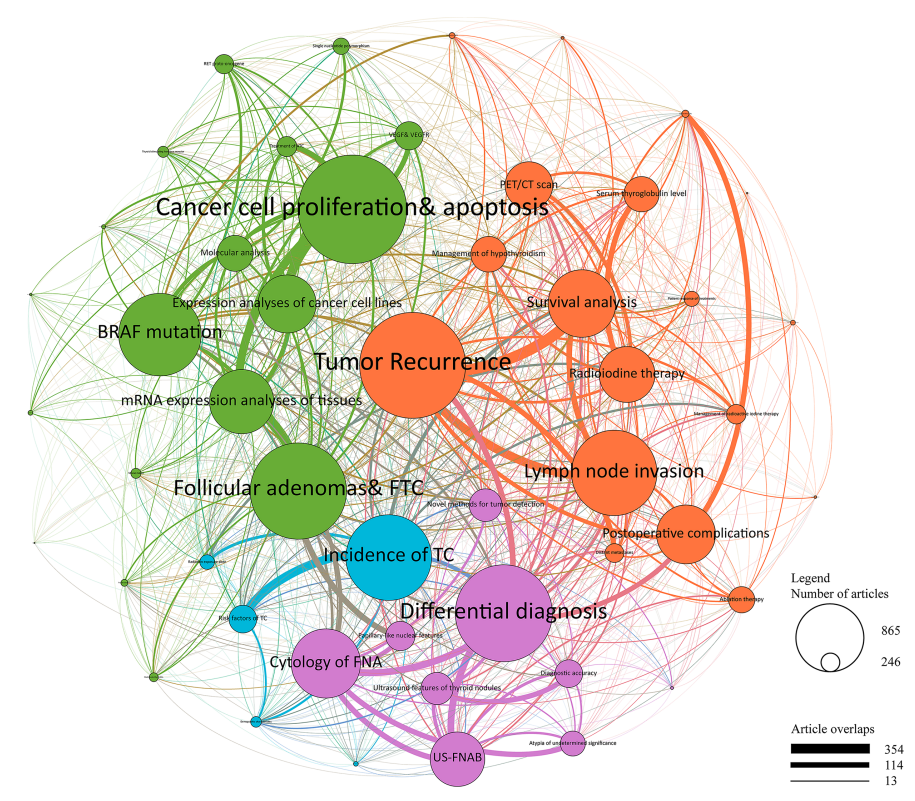

FIGURE 5 | Topic cluster network by Latent Dirichlet Allocation. Red, Treatment management; Green, Basic research; Purple, Diagnosis research; Blue, Epidemiology and cancer risk. The size of circle represents the number of papers in each topic, and the thickness of line represents the weight of connection between each topic.

TABLE 2 | Top 10 most cited publications in the field of thyroid cancer.

\begin{tabular}{|c|c|c|}
\hline Title & Journal & Citations \\
\hline $\begin{array}{l}\text { Nomenclature Revision for Encapsulated Follicular Variant of Papillary Thyroid Carcinoma: A Paradigm Shift to Reduce } \\
\text { Overtreatment of Indolent Tumors }\end{array}$ & JAMA ONCOLOGY & 723 \\
\hline Genomic and transcriptomic hallmarks of poorly differentiated and anaplastic thyroid cancers & $\begin{array}{l}\text { JOURNAL OF CLINICAL } \\
\text { INVESTIGATION }\end{array}$ & 459 \\
\hline Transoral Endoscopic Thyroidectomy Vestibular Approach: A Series of the First 60 Human Cases & WORLD JOURNAL OF SURGERY & 213 \\
\hline $\begin{array}{l}\text { Impact of reclassifying noninvasive follicular variant of papillary thyroid carcinoma on the risk of malignancy in The } \\
\text { Bethesda System for Reporting Thyroid Cytopathology }\end{array}$ & CANCER CYTOPATHOLOGY & 177 \\
\hline Natural History and Tumor Volume Kinetics of Papillary Thyroid Cancers During Active Surveillance & $\begin{array}{l}\text { JAMA OTOLARYNGOLOGY- } \\
\text { HEAD \& NECK SURGERY }\end{array}$ & 174 \\
\hline Is There a Minimum Number of Thyroidectomies a Surgeon Should Perform to Optimize Patient Outcomes? & ANNALS OF SURGERY & 168 \\
\hline $\begin{array}{l}\text { Incidences of Unfavorable Events in the Management of Low-Risk Papillary Microcarcinoma of the Thyroid by Active } \\
\text { Surveillance Versus Immediate Surgery }\end{array}$ & THYROID & 168 \\
\hline Genetic Analysis of 779 Advanced Differentiated and Anaplastic Thyroid Cancers & CLINICAL CANCER RESEARCH & 149 \\
\hline Safety and Outcomes of the Transoral Endoscopic Thyroidectomy Vestibular Approach & JAMA SURGERY & 148 \\
\hline $\begin{array}{l}\text { Vemurafenib in patients with BRAF(V600E)-positive metastatic or unresectable papillary thyroid cancer refractory to } \\
\text { radioactive iodine: A non-randomised, multicentre, open-label, phase } 2 \text { trial }\end{array}$ & LANCET ONCOLOGY & 137 \\
\hline
\end{tabular}

\section{DISCUSSION}

For the very first time, the present study comprehensively investigated 34,692 scientific publications concerning TC during the past three decades using a machine learning method. The number of publications in TC expanded rapidly, however with a small proportion of clinical trials. The most concerning topics of TC researches were thyroidectomy, differential diagnosis, genomic analysis, active surveillance. Furthermore, the results of LDA analyses also indicated a potential need for conversions of novel therapeutic targets from basic researches to treatment management. The top 10 most cited papers also covered these hot topics.

There is no doubt about the predominant role of thyroidectomy in the field of TC treatment. With the increasing incidence of TC, thyroidectomy is subsequently increasing (11). Recurrence is a critical issue when it comes to patient prognosis after thyroid surgery. A Modified Initial Risk Stratification system was proposed in the 2015 ATA guidelines, depicting the risk of recurrence in differentiated thyroid carcinoma (DTC) patients 
after initial therapy (12). Furthermore, multiple treatments are also taken to inhibit tumor recurrence, including thyroid stimulating hormone (TSH) suppression therapy and radioactive iodine (RAI) therapy (13). Another hot topic in TC treatment is lymph node invasion, as the lymph node metastases are reported in up to $50 \%$ of patients with TC (14). Lymph node invasion is proven to be associated with a higher recurrence rate and poorer survival data (15). While the lymphadenectomy is necessary for patients detected with suspicious lymph node invasion, the role of prophylactic central compartment neck dissection (pCCND) still remains controversial, mainly because of the unclear long-term outcomes of pCCND (16). In addition, novel surgical approaches, including intraoperative nerve monitoring, transoral and robotic approaches, are developing in these recent years $(17,18)$. Although unfortunately, they were not detected in MeSH term analyses or LDA analyses because of novelty, we still value their development prospects in further thyroidology.

Differential diagnosis and US-FNAB were the main topics in the diagnosis research cluster. In fact, differential diagnosis always plays as the central issue in diagnosing TC, with USFNAB as an extremely important method. US-FNAB is simple, safe, and reliable, providing the most definitive information for differential diagnosis. In most of the medical centers in the world, cytology results from US-FNAB are reporting using six categories of the 2017 updated Bethesda classification system (19). However, there are still 20 to $30 \%$ of patients with indeterminate results, requiring additional evaluations (20). As a novel diagnostic method, genomic analysis, including micro-RNA analysis, holds a great potential in contributing to the differential diagnosis (21). Future diagnosing system should take the genomic analysis into considerations. Meanwhile, the basic research cluster also showed some associations between BRAF mutation and the treatment management cluster, suggesting the potential of BRAFtargeted therapies in treating TC. However, other parts of basic research showed scarce connections with the treatment management cluster, indicating an urgent need for more conversions from basic studies to clinical treatments.

This study highlighted topics of thyroidectomy, differential diagnosis, genomic analysis, translational researches in the field of TC. Meanwhile, the top 10 most cited papers seemed to concern consistent fields. Moreover, the overtreatment issue and the active surveillance were particularly raised by most cited papers. These studies showed a large proportion of papillary thyroid cancer did not need surgical intervention. However, the problems mainly lie in effective methods to sort out the patients who need immediate surgery, and a unified standard to suggest surgery to patients who suffer from progressed lesions. The overtreatment issue may be simultaneously settled when the problems are solved. Additionally, the results of the present study showed a lack of high-quality RCT, and there was only one non-randomized study in the top 10 most cited papers. Although TC holds a relatively higher incidence than most other cancer types, most TC patients do not need any treatments other than surgery. The rest minority with advanced diseases can be further divided into several subtypes with potentially different therapeutic needs due to various pathogenic mechanisms, such as poorly differentiated TC, anaplastic TC, radioactive iodine refractory TC, etc., causing the difficulties of therapy developments, as well as a relatively small number of trial candidates in each subtype. It may be the reason for lacking high-quality researches.

There were also some limitations in this study. Firstly, only PubMed was chosen for this bibliometric research because of the high quality and comprehensive information of publications, while other databases were also available. Secondly, the $\mathrm{MeSH}$ term was used for searching publications, which might restrict the comprehensiveness of our search. Lastly, some publications were tagged with multiple publication types. Although the publication type was manually adjusted with double-check, it might still cause inaccuracy.

\section{CONCLUSION}

Thyroidectomy, differential diagnosis, genomic analysis, active surveillance are the most concerning topics in TC researches. Although the BRAF-targeted therapy is under development with promising results, there is still an urgent need for conversions from basic studies to clinical practice.

\section{DATA AVAILABILITY STATEMENT}

The original contributions presented in the study are included in the article/supplementary materials. Further inquiries can be directed to the corresponding author.

\section{AUTHOR CONTRIBUTIONS}

All authors made substantive intellectual contributions to this study to qualify as authors. XL and FX conceived the design of the study. FX modified the design of the study. ZZ, FX, WW, and LY performed the study, collected the data, and contributed to the design of the study. ZZ, LY and BJ analyzed the data. ZZ and LY drafted the Result, Discussion, and Conclusion sections. FX and WW drafted the Methods sections. FX, XL, and LY edited the manuscript. All authors read and approved the final manuscript. All authors have agreed to be accountable for all aspects of the work in ensuring that questions related to the accuracy or integrity of any part of the work are appropriately investigated and resolved.

\section{FUNDING}

This work was supported by the National Natural Science Foundation of China (grant No. 82073262) and the Hunan Province Natural Science Foundation (grant number 2019JJ40475). 


\section{REFERENCES}

1. Kim J, Gosnell JE, Roman SA. Geographic Influences in the Global Rise of Thyroid Cancer. Nat Rev Endocrinol (2020) 16(1):17-29. doi: 10.1038/ s41574-019-0263-x

2. Ma Y, Dong M, Mita C, Sun S, Peng C, Yang AC. Publication Analysis on Insomnia: How Much has Been Done in the Past Two Decades? Sleep Med (2015) 16(7):820-6. doi: 10.1016/j.sleep.2014.12.028

3. Cooper DS, Anton B. The Decade in Clinical Thyroid Disease: An Analysis of Published Literature. Thyroid (2016) 26(8):993-1003. doi: 10.1089/ thy. 2016.0018

4. Névéol A, Zweigenbaum P. Clinical Natural Language Processing in 2015: Leveraging the Variety of Texts of Clinical Interest. Yearb Med Inform (2016) 1:234-9. doi: 10.15265/IY-2016-049

5. Chen X, Xie H, Wang FL, Liu Z, Xu J, Hao T. A Bibliometric Analysis of Natural Language Processing in Medical Research. BMC Med Inform Decis (2018) 18(Suppl 1):14. doi: 10.1186/s12911-018-0594-x

6. Blei D, Carin L, Dunson D. Probabilistic Topic Models: A Focus on Graphical Model Design and Applications to Document and Image Analysis. IEEE Signal Process Mag (2010) 27(6):55-65. doi: 10.1109/MSP.2010.938079

7. Feng C, Wu Y, Gao L, Guo X, Wang Z, Xing B. Publication Landscape Analysis on Gliomas: How Much Has Been Done in the Past 25 Years? Front Oncol (2019) 9:1463. doi: 10.3389/fonc.2019.01463

8. Wang K, Feng C, Li M, Pei Q, Li Y, Zhu H, et al. A Bibliometric Analysis of 23,492 Publications on Rectal Cancer by Machine Learning: Basic Medical Research Is Needed. Therap Adv Gastroenterol (2020) 13:320843838. doi: $10.1177 / 1756284820934594$

9. Aria M, Cuccurullo C. Bibliometrix: An R-Tool for Comprehensive Science Mapping Analysis. J Informetr (2017) 4(11):959-75. doi: 10.1016/j.joi. 2017.08.007

10. Jacomy M, Venturini T, Heymann S, Bastian M. ForceAtlas2, a Continuous Graph Layout Algorithm for Handy Network Visualization Designed for the Gephi Software. PLoS One (2014) 9(6):e98679. doi: 10.1371/ journal.pone.0098679

11. Sosa JA, Hanna JW, Robinson KA, Lanman RB. Increases in Thyroid Nodule Fine-Needle Aspirations, Operations, and Diagnoses of Thyroid Cancer in the United States. Surgery (2013) 154(6):1420-6. doi: 10.1016/j.surg.2013.07.006

12. Haugen BR, Alexander EK, Bible KC, Doherty GM, Mandel SJ, Nikiforov YE, et al. 2015 American Thyroid Association Management Guidelines for Adult Patients With Thyroid Nodules and Differentiated Thyroid Cancer: The American Thyroid Association Guidelines Task Force on Thyroid Nodules and Differentiated Thyroid Cancer. Thyroid (2016) 26(1):-133. doi: 10.1089/ thy.2015.0020

13. Sohn SY, Choi JH, Kim NK, Joung JY, Cho YY, Park SM, et al. The Impact of Iodinated Contrast Agent Administered During Preoperative Computed Tomography Scan on Body Iodine Pool in Patients With Differentiated
Thyroid Cancer Preparing for Radioactive Iodine Treatment. Thyroid (2014) 24(5):872-7. doi: 10.1089/thy.2013.0238

14. Adam MA, Thomas S, Hyslop T, Scheri RP, Roman SA, Sosa JA. Exploring the Relationship Between Patient Age and Cancer-Specific Survival in Papillary Thyroid Cancer: Rethinking Current Staging Systems. J Clin Oncol (2016) 34 (36):4415-20. doi: 10.1200/JCO.2016.68.9372

15. Hughes DT, Doherty GM. Central Neck Dissection for Papillary Thyroid Cancer. Cancer Control (2011) 18(2):83-8. doi: 10.1177/ 107327481101800202

16. Wang TS, Evans DB, Fareau GG, Carroll T, Yen TW. Effect of Prophylactic Central Compartment Neck Dissection on Serum Thyroglobulin and Recommendations for Adjuvant Radioactive Iodine in Patients With Differentiated Thyroid Cancer. Ann Surg Oncol (2012) 19(13):4217-22. doi: 10.1245/s10434-012-2594-x

17. Kandil EH, Noureldine SI, Yao L, Slakey DP. Robotic Transaxillary Thyroidectomy: An Examination of the First One Hundred Cases. J Am Coll Surg (2012) 214(4):558-64. doi: 10.1016/j.jamcollsurg.2012.01.002

18. Anuwong A. Transoral Endoscopic Thyroidectomy Vestibular Approach: A Series of the First 60 Human Cases. World J Surg (2016) 40(3):491-7. doi: 10.1007/s00268-015-3320-1

19. Cibas ES, Ali SZ. The 2017 Bethesda System for Reporting Thyroid Cytopathology. Thyroid (2017) 27(11):1341-6. doi: 10.1089/thy.2017.0500

20. Nikiforov YE. Role of Molecular Markers in Thyroid Nodule Management: Then and Now. Endocr Pract (2017) 23(8):979-88. doi: 10.4158/EP171805.RA

21. Lithwick-Yanai G, Dromi N, Shtabsky A, Morgenstern S, Strenov Y, Feinmesser M, et al. Multicentre Validation of a microRNA-Based Assay for Diagnosing Indeterminate Thyroid Nodules Utilising Fine Needle Aspirate Smears. J Clin Pathol (2017) 70(6):500-7. doi: 10.1136/jclinpath2016-204089

Conflict of Interest: The authors declare that the research was conducted in the absence of any commercial or financial relationships that could be construed as a potential conflict of interest.

Publisher's Note: All claims expressed in this article are solely those of the authors and do not necessarily represent those of their affiliated organizations, or those of the publisher, the editors and the reviewers. Any product that may be evaluated in this article, or claim that may be made by its manufacturer, is not guaranteed or endorsed by the publisher.

Copyright $\odot 2021$ Zhang, Yao, Wang, Jiang, Xia and Li. This is an open-access article distributed under the terms of the Creative Commons Attribution License (CC BY). The use, distribution or reproduction in other forums is permitted, provided the original author(s) and the copyright owner(s) are credited and that the original publication in this journal is cited, in accordance with accepted academic practice. No use, distribution or reproduction is permitted which does not comply with these terms. 